\title{
Influência do ciclo lunar no parto: mito ou constatação científica?
}

\author{
Influences of lunar cycle in labor: myth or scientific finding? \\ Influencia del ciclo lunar en el parto: mito o constatación científica?
}

Aline Bueno', Isabela Lovizutto lessi', Débora Cristina Damasceno'

'Universidade Estadual de São Paulo. Faculdade de Medicina de Botucatu. Departamento de Ginecologia e Obstetrícia. Botucatu, SP

Submissão: 12/12/2008

Aprovação: 09/02/2010

\section{RESUMO}

Muitas superstições são encontradas em nossas vidas. Na medicina, uma profissão Que se orgulha em métodos baseados em evidências para tratamentos, isso não é uma exceção. Uma superstição que abrange o parto é a influência de determinadas fases do ciclo da lua, mais especificamente a lua cheia. Embora estudos tenham demonstrado que o aumento de partos está relacionado com o ciclo lunar, existe discordância sobre Quando ocorre o pico do volume de partos em cada fase da lua. Frente à divergência de resultados existentes na literatura Que relaciona os eventos do ciclo lunar com parto, o objetivo desta revisão foi realizar um levantamento bibliográfico na tentativa de esclarecer esta cultura popular com base nos resultados apresentados por diferentes autores.

Descritores: Ciclo lunar; Parto; Enfermagem obstétrica.

\section{ABSTRACT}

Superstitions are found everywhere in our lives, and medicine, a profession that is prides itself on an evidence-based approach to treatment, is not exempt. A superstition that pervades the labor and delivery floor is that it is busier during certain phases of the lunar cycle, specifically the full moon. Although some studies have demonstrated an increase in deliveries that are related to the lunar cycle, there has been disagreement about when, in the lunar cycle, the peak volume occurs. Front to the divergence of the existent results in the literature to relate the events of the lunar cycle with deliveries, the aim of this review was to accomplish the literature in the attempt of explaining this popular culture with base in the results presented by different researchers.

Key words: Moon; Delivery; Obstetrical nursing.

\section{RESUMEN}

Se encuentran las supersticiones por todas partes en nuestras vidas, y la medicina, una profesión Que tiene orgullo acerca de los tratamientos con base en evidencia, no está extinta. La influencia de ciertas fases del ciclo lunar, específicamente la luna llena, es una superstición sobre el parto. Auneue algunos estudios han demostrado un aumento en los partos Que se relacionan al ciclo lunar, ha habido discordancia sobre cuando, en el ciclo lunar, el volumen máximo ocurre. Afronte a la divergencia de los resultados existentes en la literatura Que relacionan los eventos del ciclo lunar con los partos, el objetivo de esta revisión era explorar la literatura en el esfuerzo de explicar esta cultura popular con la base en los resultados presentado por los diferentes investigadores.

Descriptores: Luna; Parto; Enfermería obstétrica. 


\section{INTRODUÇÃO}

Muitas superstições são encontradas ao longo de nossas vidas. Na medicina, uma profissão Que se orgulha em métodos baseados em evidências para tratamentos, isso não é uma exceção. Uma superstição Que abrange o parto é a influência de determinadas fases do ciclo lunar, mais especificamente a lua cheia ${ }^{(1)}$.

Em diferentes culturas e mitologias, a lua está relacionada com fertilidade, gestação e parto. Obstetras profissionais também notam aumento na demanda nos dias em que a lua é cheia. Diversos estudos têm sido feitos na tentativa de correlacionar o número de parto com as fases da lua, mas os resultados são contraditórios ${ }^{(2)}$. A relação entre lua cheia e comportamento humano tem sido considerada há muito tempo e uma variedade de estudos tem sido publicada, por vezes, com resultados divergentes ${ }^{(3)}$.

A idéia de que a meteorologia afeta o aparecimento do parto criou raízes na cultura popular. Os primeiros calendários foram baseados no ciclo lunar. A agricultura foi desenvolvida há longo tempo baseada nas fases da lua. Com o aumento da civilização, a lua ganhou maior importância. As mulheres são tão influenciadas pela lua Que muitas acreditavam que a fertilidade e o parto variavam de acordo com a fase lunar ${ }^{(4)}$.

Segundo Ghiandoni et al ${ }^{(5)}$, há evidências de Que o magnetismo terrestre, a luz lunar e a atração gravitacional da lua sob a terra são fatores Que podem alterar o tempo e o tipo de parto.

Embora alguns estudos tenham demonstrado Que o aumento de partos esteja relacionado ao ciclo lunar, existe discordância sobre Quando ocorre o pico do volume de partos em cada fase lunar. Os pesquisadores discordam ainda, Quanto à Questão de saber se um pico ocorre para todos os partos ou para um subconjunto de partos (tais como as mulheres multíparas e partos vaginais) ou ainda, se o pico é para o início do trabalho de parto. Do mesmo modo, existem dúvidas Quanto à Questão de saber se o pico diz respeito à frequência de partos ou de complicações ${ }^{(1)}$.

Objetiva-se, com este estudo, apresentar uma breve revisão da literatura acerca da hipótese de que o trabalho de parto e a suas possíveis complicações sofrem influência das fases da lua, de modo a propor uma reflexão sobre as implicações advindas destas intercorrências. Acredita-se Que os achados de tal investigação possam contribuir com o entendimento do assunto, lançando subsídios teóricos para os profissionais na área de enfermagem, obstetrícia e emergência visando um preparo diferenciado em determinados dias em oue, possivelmente, a fase da lua possa influenciar a demanda de partos ou suas complicações facilitando a elaboração de estratégias de acompanhamento e aprimoramento dessa modalidade de cuidado.

\section{MÉTODO}

Para este estudo, foi realizada revisão de literatura referente ao período de 1975 a 2008 no site do database do National Center of Biotechnology Information (NCBI - PUBMED) e no Scielo. Foram empregados os termos: delivery and moon and/or lunar cycle and/or lunar phase.

\section{RESULTADOS E DISCUSSÃO}

Na tentativa de confirmar a influência da lua cheia na hora do parto e a relação observável e fácil entre parto e fases da lua, considerando especialmente o pico na lua cheia, Schwab ${ }^{(3)}$ selecionou 4.836 nascimentos durante 731 dias, sendo Que 174 ocorreram em 24 dias de lua cheia. Após várias análises, o autor concluiu Que novos estudos deveriam ser realizados com técnicas estatísticas mais sofisticadas para refinar mais os dados a fim de se confirmar se a lua cheia realmente interfere no pico de número de partos.

Outro estudo para esclarecer a dúvida sobre a influência da lua na hora do parto avaliou a distribuição de todos os nascimentos durante 51 ciclos lunares no período de março de 1974 a abril de 1978 no Hospital Universitário da Califórnia. Houve 11.691 nascidos vivos, dos Quais 8.142 foram naturais, 141 múltiplos e 168 natimortos. Em nenhuma das Quatro amostras, o número de nascimentos ocorreu na lua cheia, mostrando eue a taxa de nascimento durante o período estudado não se correlacionou com o ciclo da fase lunar ${ }^{(6)}$.

De acordo com Strolego ${ }^{(7)}$, diversos fatores alteram a data do parto, atrasando ou adiantando o nascimento. Algumas culturas acreditam Que determinadas fases da lua influenciam mais do Que outras na hora do parto. Para validar a crença, o estudo foi realizado dentro de 37 ciclos, envolvendo 5.226 partos. O resultado não mostrou diferença estatística entre a fase de lua cheia e as demais fases do ciclo, mesmo considerando dois dias antes e dois dias após a mudança da fase lunar, sugerindo Que essa influência é improvável.

Ong et $\mathrm{al}^{(8)}$ também investigaram a cultura popular de Que o ciclo lunar afeta o trabalho de parto. Foram selecionados 10.027 partos no período de 18 meses. Não houve aumento no número de partos durante as fases de lua cheia comparado às outras fases. As taxas de cesáreas, partos vaginas e partos prematuros não se alteraram em função da lua cheia. Esses autores concluíram Que o ciclo lunar não influenciou na atividade do parto.

Por outro lado, estudo demonstrou a relação entre a posição da lua, o dia do parto e a distribuição de partos espontâneos, especialmente na presença de lua cheia. A análise realizada incluiu gestantes a termo ( 38 a 42 semanas de gestação) resultando em 1.248 partos espontâneos num período de 3 anos dentro de 36 ciclos lunares. De acordo com uma análise estatística mais específica e aplicada, os resultados mostraram Que há conexão entre a distribuição de partos espontâneos e o ciclo lunar e Que este efeito parece relevante, principalmente em multíparas ${ }^{(5)}$.

Investigações deste tipo continuaram a ser realizadas, dentre elas, Romero et $\mathrm{al}^{(2)}$ relacionaram todas as fases do ciclo lunar com a distribuição dos nascimentos para descobrir se as fases da lua influenciam na distribuição dos partos e se de fato há confirmação da cultura popular. O estudo teve um total de 1.715 partos completando 10 ciclos lunares. As gestantes foram divididas em multíparas e nulíparas e a diferença encontrada na distribuição de partos entre as fases do ciclo lunar junto com a comparação do numero de partos no dia central de cada fase não mostrou diferença estatisticamente significativa.

Arliss et $\mathrm{al}^{(1)}$ avaliaram o efeito do ciclo lunar na frequência de partos, e o mesmo efeito nas complicações mais frequentes da hora do parto. A comparação foi feita entre 564.039 partos totalizando 62 ciclos lunares, considerando oito fases da lua (nova, tornando-se crescente, crescente, primeiro Quarto, último Quarto, tornando-se minguante, cheia e minguante) no período de 1997 - 
2001. Os autores não verificaram diferença entre o numero de nascimentos Que ocorreram durante as oito fases da lua para todos os nascimentos (induzidos, não-induzidos, de mulheres multíparas ou Que tiveram parto vaginal).

Foi realizada uma investigação da influência da lua nova e da lua cheia no trabalho de parto e na ruptura espontânea de membrana considerando a pressão barométrica. O estudo ocorreu no ano de 1984 em Cleveland (Ohio) e foram inclusos partos espontâneos ou com ruptura de membrana, Que totalizaram um número amostral de 1999. A pressão barométrica foi mensurada diariamente e os dias de lua cheia e nova foram anotados. A freeuência de partos espontâneos e de ruptura espontânea de membranas na lua nova e na lua cheia foi comparada com os dias controlados pela pressão barométrica. Os resultados mostraram correlação significativa na frequência de partos na lua cheia Quando a pressão barométrica era descontrolada. No entanto, Quando a pressão era controlada, não houve correção positiva significativa na frequência de partos e ruptura espontânea de membranas ${ }^{(9)}$.

\section{Considerações finais}

Embora sejam encontradas variações consideráveis na fręuência de partos dentro das fases da lua, não há evidências estatísticas Que comprovem esta relação, exceto por um trabalho científico. Apesar de nossos achados, acreditamos Que a superstição entre lua e aumento do número de partos continuará a existir.

\section{REFERÊNCIAS}

1. Arliss JM, Kaplan EN, Galvin SL. The effect of the lunar cycle on freeuency of births and birth complications. Am I Obstet Gynecol 2005; 192: 1462-4.

2. Romero MI, Guerreiro GI, Artura AS. The moon and delivery. Rev Enferm 2004; 27: 7-9.

3. Schwab B. Letter: Delivery of babies and the full moon. Can Med Assoc I 1975; 113: 489-93.

4. Morton-Pradhan S, Bay RC, Coonrod DV. Birth rate and its correlations with the lunar cycle and specific atmospheric conditions. Am J Obstet Gynecol 2005; 192: 1970-3.

5. Ghiandoni G, Seclì R, Rocchi MBL, Ugolini G. Does lunar position influence the time of delivery? A statistical analysis.
Eur J Obstet Gynecol Reprod Biol 1998; 77: 47-50.

6. Abell GO, Greenspan B. Human births and the phase of the moon. N Engl I Med 1979; 300: 96.

7. Strolego F, Gigli C, Bugalho A. The influence of lunar phases on the frequency of deliveries. Minerva Ginecol 1991; 43: 359-63.

8. Ong S, Wingfield M, McQuillan K. Labour ward activity and the lunar cycle. I Obstet Gynaecol 1998; 18: 538-9.

9. Stern EW, Glazer GL, Sanduleak N. Influence of the full and new moon on onset of labor and spontaneous rupture of membranes. I Nurse Midwifery 1988; 33: 57-61. 